\title{
TOTAL INFORMATION AWARENESS (DARPA'S RESEARCH PROGRAM)
}

\begin{abstract}
Tn response to September 11, 2001, the Defense Advanced Research Projects Agency (DARPA) created the Information Awareness Office (IAO) to research, develop, and demonstrate innovative information technologies to detect terrorist groups planning attacks against American citizens, anywhere in the world. ${ }^{1}$ DARPA's mission is to research and demonstrate innovative technologies to solve national-level problems such as the grave terrorist threat. The mission of IAO is to imagine, develop, apply, integrate, demonstrate, and transition information technologies, components, and prototype closed-loop information systems that will counter asymmetric threats by achieving total information awareness in support of preemption, national security warning, and national security decision making.
\end{abstract}

The Total Information Awareness (TIA) is the main program of IAO beginning in fiscal year 2003. The stated goal of the program is to "revolutionize the ability of the United States to detect, classify and identify foreign terrorists - and decipher their plans - and thereby enable the U.S. to take timely action to successfully preempt and defeat terrorist acts." ${ }^{2}$ The TIA objective is to create a counter-terrorism information system that: (1) increases information coverage and affords easy future scaling; (2) provides focused warnings within an hour after a triggering event occurs or an evidence threshold is passed; (3) can automatically queue analysts based on partial pattern matches in a patterns' database that covers 90 percent of all known terrorist attacks; and, (4) supports collaboration, analytical reasoning and information sharing so that analysts can hypothesize, test and propose theories and mitigating strategies about possible futures, so decision-makers can effectively evaluate the impact of current or future policies and prospective courses of action.

Figure 1 graphically presents the TIA vision. The strategy is to integrate appropriate technologies into a series of increasingly powerful prototype systems to be tested in operationally relevant environments, using real-time feedback to refine concepts of operation and performance requirements. The program is focusing on the development of architectures for a large-scale counter-terrorism database, for system elements associated with database population, and for integrating algorithms and mixed-initiative analytical tools; novel methods for populating the database from 
existing sources, create innovative new sources, and invent new algorithms for mining, combining, and refining information for subsequent inclusion into the database; revolutionary new models, algorithms, methods, tools, and techniques for analyzing and correlating information in the database to derive actionable intelligence.

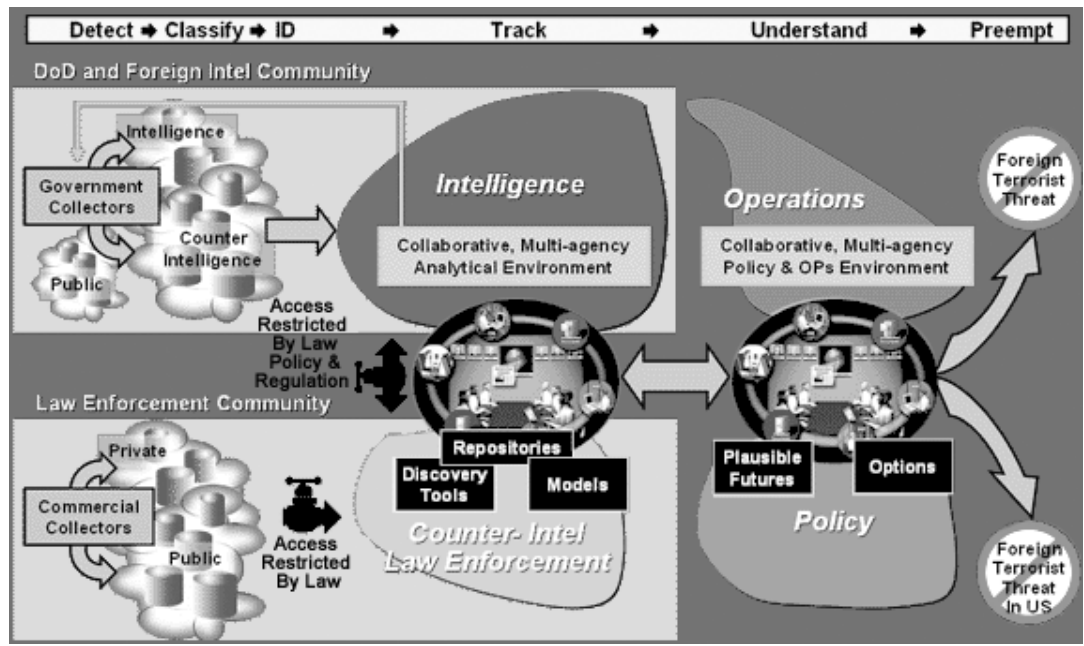

Figure 1: Overview of the Total Information Awareness system.

TIA is an overarching program paralleled by a dozen of technology programs. ${ }^{3}$ Three of these deal with language processing challenges. The goal of the Babylon program is to develop rapid, two-way, natural language speech translation interfaces and platforms for the warfighter and shall be used in field environment. The Translingual Information Detection, Extraction and Summarization (TIDES) program is developing advanced language processing technology to enable English speakers to find and interpret critical information in multiple languages without requiring knowledge of those languages. The Effective Affordable Reusable Speech-To-Text $(E A R S)$ program is developing automatic speech-to-text transcription technology whose output is substantially richer and much more accurate than currently possible. The program seeks to develop a robust technology with an error rate of five to ten percent for broadcast and conversational speech. ${ }^{4}$ The expectation is that machines will do a much better job of detecting, extracting, summarizing, and translating important information thus allowing humans to understand what was said by reading transcripts instead of listening to audio signals.

A related program called Communicator is expected to develop and demonstrate "dialogue interaction" technology that enables warfighters to talk with computers. 
The objective is to make information accessible on the battlefield or in command centers without ever having to touch a keyboard. The Communicator Platform will be wireless and mobile, and will function in a networked environment. Software enabling dialogue interaction will automatically focus on the context of a dialogue to improve performance, and the system shall be capable of automatically adapting to new topics so conversation is natural and efficient.

A number of programs deal with information storage and processing challenges. The goal of the program Genisys is to produce technology enabling ultra-large, all-source information repositories. In order to predict, track, and preempt terrorist attacks, the U.S. requires a full-coverage database containing all information relevant to identifying potential foreign terrorists and possible supporters, their activities, prospective targets, and operational plans.

The Evidence Extraction and Link Discovery (EELD) program is developing technologies and tools for automated discovery, extraction and linking of sparse evidence contained in large amounts of classified and unclassified data sources. EELD is expected to provide detection capabilities to extract relevant data and relationships about people, organizations, and activities from message traffic and open source data. It will link items relating potential terrorist groups or scenarios, and learn patterns of different groups or scenarios to identify new organizations or emerging threats.

Project Genoa, which is being finalized, provides structured argumentation, decisionmaking and corporate memory to rapidly deal with and adjust to dynamic crisis management. It creates virtual collaborative environment for both analyst and policy making communities (Figure 2). The Genoa II program was launched in FY03. It will focus on developing information technology needed by teams of intelligence analysts and operations and policy personnel in attempting to anticipate and preempt terrorist threats to US interests. The goal is to make such teams faster, smarter, and more joint in their day-to-day operations. Genoa II will apply automation to team processes so that more information will be exploited, more hypotheses created and examined, more models built and populated with evidence. As a result, it is expected that teams will be able to deal with more crises simultaneously.

Advances in decision-making are supported by modeling terrorist behavior in the Wargaming the Asymmetric Environment (WAE) program. Its goal is to develop and demonstrate predictive technology to better anticipate and act against terrorists. WAE is a revolutionary approach to identify predictive indicators of attacks by and the behavior of specific terrorists by examining their behavior in the broader context of their political, cultural and ideological environment. 


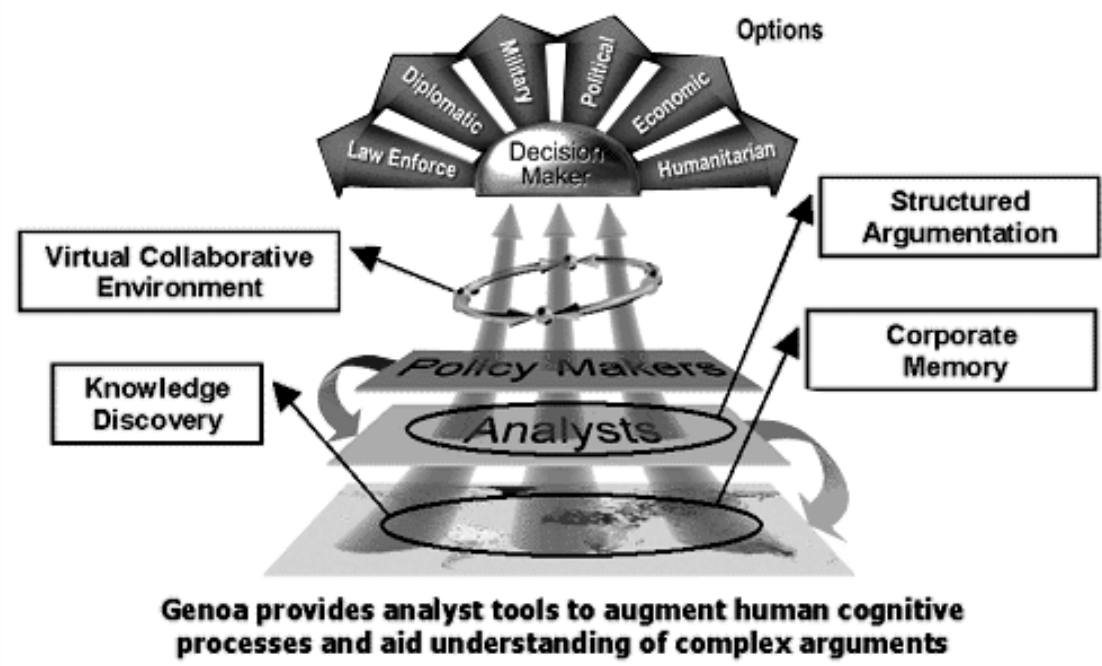

Figure 2: Structured argumentation and decision-making environment.

TIA is expected to benefit also from related business technology developments. The Futures Markets Applied to Prediction (FutureMAP) program is a follow-up to a current DARPA program named Electronic Market-Based Decision Support. FutureMAP will concentrate on market-based techniques for avoiding surprise and predicting future events. Strategic decisions depend upon the accurate assessment of the likelihood of future events. This analysis often requires independent contributions by experts in a wide variety of fields, with the resulting difficulty of combining the various opinions into one assessment. Market-based techniques provide a tool for producing these assessments. Potential applications may include analysis of political stability in regions of the world, prediction of the timing and impact on national security of emerging technologies, analysis of the outcomes of advanced technology programs, etc. In addition, the rapid reaction of markets to knowledge held by only a few participants may provide an early warning system to avoid surprise.

TIA further incorporates bio-surveillance programs designed to provide early warning and detection of incidents of bioterrorism. The goal of the Bio-event Advanced Leading Indicator Recognition Technology (Bio-ALIRT) program is to develop the necessary information technologies and resulting prototype capable of detecting the covert release of a biological pathogen automatically and significantly earlier than traditional approaches. Early detection is considered the key to mitigating a biological attack. Given the availability of appropriate medications, as many as half the expected casualties could be prevented if an attack is detected only a few days earlier than it would have otherwise been identified. For contagious biological agents, 
early detection is also clearly paramount. The Bio-ALIRT program shall dramatically increase the ability to detect a clandestine biological warfare attack in time to respond effectively and to avoid potentially thousands of casualties.

Finally, the Human Identification at a Distance (HumanID) program aims at developing automated biometric identification technologies to detect, recognize and identify humans at great distances. These technologies are expected to provide critical early warning support for force protection and homeland defense against terrorist, criminal, and other human-based threats, and to prevent or decrease the success rate of such attacks against operational facilities and installations of the Department of Defense. Methods for fusing biometric technologies into advanced human identification systems will be developed to enable faster, more accurate and unconstrained identification of humans at significant standoff distances.

The research conducted under TIA will provide the tools for obtaining information pertaining to activities of terrorists, and if connected together, this information could alert authorities before terrorists' plans are carried out. Overall, the development of these anti-terrorism tracking tools is expected to allow the agencies to better execute their missions. While the research to date is promising, TIA is still only a concept. ${ }^{5}$

TIA is not collecting or gathering any intelligence information. The US Government confirms that this is and will continue to be the responsibility of the US foreign intelligence/ counterintelligence agencies, which operate under various legal and policy restrictions with congressional oversight. This technology development program does not alter the authority or responsibility of the intelligence community. TIA is a research program designed to catch terrorists before they strike.

Information \& Security

\section{Notes:}

The Information Awareness Office at http://www.darpa.mil/iao/index.htm. http://www.darpa.mil/iao/TIASystems.htm.

Described also in "Researchers Leave Terrorists Nowhere to Hide," SIGNAL 57, 6 (February 2003): 43-46.

4 "Researchers Leave Terrorists Nowhere to Hide," p. 45.

“Total Information Awareness (TIA) Update," DefenseLINK No. 060-03 (February 7, 2003), <http://www.defenselink.mil/news/Feb2003/b02072003_bt060-03.html>. 